\title{
The Beneficial Effects of Alpha Lipoic Acid Supplementation on Lp-PLA2 Mass and Its Distribution between HDL and apoB- Containing Lipoproteins in Type 2 Diabetic Patients: A Randomized, Double-Blind, Placebo-Controlled Trial
}

\author{
Nima Baziar $\mathbb{D}^{1},{ }^{1}$ Ensieh Nasli-Esfahani, ${ }^{2}$ Kurosh Djafarian, ${ }^{1}$ Mostafa Qorbani $\mathbb{D}^{3,4}$ \\ Mehdi Hedayati $\odot{ }^{5}{ }^{5}$ Mahshid Abd Mishani $\odot{ }^{6},{ }^{6}$ Zeinab Faghfoori, ${ }^{7}$ Najva Ahmaripour, ${ }^{5}$ \\ and Saeed Hosseini ${ }^{1}{ }^{1}$ \\ ${ }^{1}$ Department of Clinical Nutrition, School of Nutritional Sciences and Dietetics, Tehran University of Medical Sciences, Tehran, Iran \\ ${ }^{2}$ Diabetes Research Center, Endocrinology and Metabolism Clinical Sciences Institute, Tehran, Iran \\ ${ }^{3}$ Non-Communicable Diseases Research Center, Alborz University of Medical Sciences, Karaj, Iran \\ ${ }^{4}$ Chronic Diseases Research Center, Endocrinology and Metabolism Population Sciences Institute, Endocrinology and Metabolism \\ Research Institute, Tehran University of Medical Sciences, Tehran, Iran \\ ${ }^{5}$ Cellular and Molecular Endocrine Research Center, Research Institute for Endocrine Sciences, Shahid Beheshti University of \\ Medical Sciences, Tehran, Iran \\ ${ }^{6}$ Department of Clinical Nutrition, School of Nutritional and Food Industry, Shahid Beheshti University of Medical Sciences, \\ Tehran, Iran \\ ${ }^{7}$ Food Safety Research Center (salt), Semnan University of Medical Sciences, Semnan, Iran
}

Correspondence should be addressed to Saeed Hosseini; saeedhmdphd@hotmail.com

Received 11 October 2019; Accepted 11 January 2020; Published 10 March 2020

Guest Editor: Fiona L. Wilkinson

Copyright (c) 2020 Nima Baziar et al. This is an open access article distributed under the Creative Commons Attribution License, which permits unrestricted use, distribution, and reproduction in any medium, provided the original work is properly cited.

\begin{abstract}
Lipoprotein-associated phospholipase $A_{2}$ (Lp-PLA2) is a new specific vascular inflammation biomarker that is carried by the lipoproteins in the blood and plays a prominent role in the pathogenesis of atherosclerosis. Increased Lp-PLA2 levels and impaired Lp-PLA2 distribution across high-density lipoprotein (HDL) and non-HDL lipoproteins have been reported in diabetic patients, which is associated with the increase in cardiovascular disease (CVD) risk. This study is aimed at investigating the effect of alpha lipoic acid (ALA), as an antioxidant with potential cardioprotective properties, on the Lp-PLA2 mass and its distribution in diabetic patients. In a double-blind, randomized, placebo-controlled clinical trial, seventy diabetic patients were randomly allocated to ALA (1200 mg ALA as two $600 \mathrm{mg}$ capsules/day) and placebo (two maltodextrin capsules/day) groups. The serum levels of total Lp-PLA2 mass, HDL-Lp-PLA2, oxidized low-density lipoproteins (ox-LDL), apolipoprotein A1 (apo A1), lipid profiles, fasting blood sugar (FBS), and insulin were measured, and apolipoprotein B- (apoB-) associated Lp-PLA2 and homeostasis model of assessment index (HOMA-IR) were calculated at the baseline and after 8 weeks of intervention. ALA significantly decreased the ox-LDL, total Lp-PLA2 mass, apoB-associated Lp-PLA2, and percent of apoB-associated Lp-PLA2 and triglyceride and increased the percent of HDL-Lp-PLA2 compared with the placebo group but had no significant effect on HDL-Lp-PLA2 mass, apo A1, lipid profiles, and glycemic indices. There was a positive correlation between the reduction in the ox-LDL level and total Lp-PLA2 mass in the ALA group. In conclusion, ALA may decrease the CVD risk by reducing the oxLDL and Lp-PLA2 mass and improving the Lp-PLA2 distribution among lipoproteins in type 2 diabetic patients.
\end{abstract}




\section{Introduction}

Type 2 diabetic (T2D) patients present a high prevalence of cardiovascular events with or without established cardiovascular diseases (CVDs) [1]. The underlying mechanisms in the development of CVD in patients with diabetes mellitus (DM) are complex, and it seems inflammatory processes and oxidative stress play a vital role in the pathogenesis of both of them $[2,3]$.

Of all the recognized inflammatory markers, lipoproteinassociated phospholipase $\mathrm{A}_{2}$ (Lp-PLA2) is a novel specific vascular inflammation and atherosclerosis biomarker that is secreted by macrophages and bound to lipoproteins in the bloodstream [4]. According to Adult Treatment Panel-III (ATP-III) guidelines, measuring the Lp-PLA2 levels is useful for a more accurate diagnosis of coronary heart disease (CHD) risk [5], especially in patients with low-density lipoprotein (LDL) less than $130 \mathrm{mg} / \mathrm{dl}$ [6]. A Lp-PLA2 level higher than $200 \mathrm{ng} / \mathrm{ml}$ represents that the patient is at a higher risk [5].

Lp-PLA2 may have a role in the pathogenesis of atherosclerosis. This enzyme breakdowns phospholipids in oxidized low-density lipoproteins (ox-LDL) into two proinflammatory and proatherogenic products, lysophosphatidylcholine and oxidized free fatty acids, these inflammatory factors promote atherosclerosis [7-9]. Therefore, it has been proposed that Lp-PLA2 mediates ox-LDL-induced inflammatory responses in the atherosclerotic plaque and is a predictor of coronary events independent of traditional risk factors $[4,8,10]$. However, recently, Lp-PLA2 inhibitor darapladib in two phase III clinical trials in patients with acute coronary syndrome or CHD did not have a protective role in the prevention of further major vascular disease. Indeed, based on several genome-wide association studies (GWAS), the causal relation between Lp-PLA2 and CHD remains controversial [11-13].

Some studies have found that Lp-PLA2 and ox-LDL levels in diabetic patients are higher than those in healthy individuals [14]. Higher levels of this enzyme are associated with the increased risk of incident of CHD among diabetic patients, so Lp-PLA2 could be considered a potential therapeutic target to decrease atherosclerotic risk and the development of cardiometabolic complications [7, 15, 16]. Given the proposed role of ox-LDL in the production of Lp-PLA2, it has been suggested that reducing Lp-PLA2 mass by oxLDL-lowering intervention may decrease the risk of CVD in diabetic patients [17-19].

On the other hand, it has been proposed that Lp-PLA2 may have both proatherogenic and antiatherogenic features depending on the type of lipoprotein which this enzyme is bound. About $70 \%$ to $80 \%$ of the total serum Lp-PLA2 bound to LDL, whereas the $20 \%$ to $30 \%$ is associated with highdensity lipoproteins (HDL) $[15,20]$. It is assumed that HDL-associated Lp-PLA2 has an anti-inflammatory and vasculoprotective activity, whereas Lp-PLA2 bound to apoB lipoproteins is proinflammatory and atherogenic. Therefore, the relative distribution of Lp-PLA2 could be useful in the assessment of CVD risk [21,22]. An altered distribution of Lp-PLA2, with a proportionately less mass of HDL, has been reported in diabetic patients [23].
Alpha lipoic acid (ALA), also known as thioctic acid, as an essential cofactor of dehydrogenase enzymes, is involved in mitochondrial macronutrient metabolism. ALA has antioxidant characteristics that unlike most other antioxidants, is both water- and fat-soluble; thus, it can reach easily into tissues composed of fat, such as the nervous system as well as those mainly of water such as the cardiovascular system, so considered a "universal antioxidant" [24-26]. ALA exhibits direct free radical scavenging properties and with respect to lower redox potential $(-0.32 \mathrm{v})$ compared to many antioxidants, such as carotenoids, vitamin E, vitamin C, and glutathione, and has more antioxidant potency and so, it is able to regenerate these antioxidants [24, 27-29]. ALA has beneficial effects in preventing or relieving symptoms of diseases related to oxidative stress such as diabetes and cardiovascular disease [25, 30-32]. Animal and in vitro studies have suggested antiatherogenic properties for ALA; however, few clinical studies have been conducted in this regard [33, 34]. ALA may protect against atherosclerotic cardiovascular diseases (ASCVD) by inactivating reactive oxygen species (ROS) and chelating metal ion, subsequently preventing LDL oxidation and improving lipoprotein metabolism [35-37].

In in vitro studies, induction of oxidative stress via oxLDL and ROS has been associated with increased Lp-PLA2 gene expression in human monocytic cells (THP-1). On the other hand, it has been shown that ALA suppressed ROSstimulated Lp-PLA2 gene expression in this immune cell $[19,38]$. Thus, we hypothesized that ALA can reduce the Lp-PLA2 mass and its distribution among HDL and apoBcontaining lipoproteins in diabetic patients.

To the best of our knowledge, no intervention study has examined the effect of ALA on Lp-PLA2 mass and its distribution among lipoproteins in type 2 diabetes mellitus patients. Therefore, this interventional study is aimed at investigating the effects of ALA supplementation on Lp-PLA2 mass and its distribution between HDL and apoB-containing lipoproteins and ox-LDL in type 2 diabetes mellitus.

\section{Materials and Methods}

2.1. Participants. This study was conducted from May to August 2019 at the Diabetes and Metabolic Disease Clinic of Endocrinology and Metabolism Research Institute, Tehran University of Medical Sciences. T2D patients who were willing to participate in the study were invited based on their medical records for the last three months. After assessing for eligibility, 70 non-insulin-dependent diabetes mellitus (NIDDM) patients with a body mass index (BMI) between 18.5 and 29.9, aged between 40 and 60 years old, diagnosis of Type $2 \mathrm{DM}$ for at least two years, and $\mathrm{HbA} 1 \mathrm{C}<7 \%$ were enrolled in the study.

The following were criteria for the exclusion of subjects from the study: (a) history of heart attack, angina pectoris, stroke, and other chronic diseases and contagious diseases in the past year; (b) use of smoke and alcohol during the last three months prior the study; (c) changing in medications received in the previous three months; (d) using any drug other than metformin, sulfonylurea, statins, angiotensinconverting enzyme inhibitors (ACEI), angiotensin receptor 
blockers (ARB), and aspirin at doses of 80 mg or less; (e) taking any supplements containing antioxidants and omega- 3 in the past three months (at least once a week); and (f) pregnancy and breastfeeding. Also, the exclusion criteria during the study were the unwillingness to continue the cooperation, change in any of the entry criteria, and possible side effects of taking supplements and nonregular consumption of supplements (less than $80 \%$ of the supplements).

The aim and procedure of the study were described to each subject, and the voluntary written informed consent was signed before the initiation of the trial. This clinical trial was registered with the Iranian Registry of Clinical Trials (https://www.irct.ir/ trial/30483, identifier: IRCT20180407039219N1. Registered on 27 April 2018) and was approved by the ethical committee of Tehran University of Medical Sciences, Tehran, Iran (reference number IR.TUMS.VCR.REC.1396.4583).

The sample size was calculated based on a significant mean difference of Lp-PLA2, which as observed in previous studies [39], with a $95 \%$ confidence level and $80 \%$ power, a total of 32 subjects were estimated for each group. Assuming the $10 \%$ dropout rates, the sample size was increased in 35 subjects in each group.

2.2. Study Design and Intervention. This study is a doubleblind, randomized, parallel, placebo-controlled clinical trial. Seventy diabetic patients were randomly allocated in a ratio of $1: 1$ into the intervention and control groups by permuted block randomization. We used stratified randomization to match participants based on sex and menopausal in both groups.

Subjects in the intervention group $(n=35)$ and the control group ( $n=35$ ) received $1200 \mathrm{mg}$ of ALA supplement per day (as two $600 \mathrm{mg}$ capsules) and placebo capsules (maltodextrin), respectively, for eight weeks. In order to better absorption and minimize possible gastrointestinal complications, all patients were asked to take one capsule, two times daily 30 minutes before meals. The shape, odor, taste, and packaging of the supplements and placebo capsules were similar. For blinding the researcher and patients, the capsules' containers were marked with A and B by an expert in the clinic who was blind to the study. ALA supplements (Support Nutrition Company, USA) are supplied by Darou Darman Sepehr Co.

For improving the patients' adherence to the study, we called them up and reminded taking capsules; also, we asked about possible side effects such as skin sensation, fever, and gastrointestinal discomfort. Due to difficulty in measuring the ALA concentration, for evaluating the patients' compliance, the remaining capsules were counted at the $4^{\text {th }}$ and $8^{\text {th }}$ week. We asked the participants to continue their routine lifestyle, including dietary and physical activity habits during the study and notify us in case of changing the lifestyle or drugs for any reason.

We measured primary outcomes including the Lp-PLA2 mass, the percent of HDL-Lp-PLA2, and the percent of apoB-associated Lp-PLA2 and secondary outcomes including the fasting serum levels of ox-LDL, insulin, glucose, triglyceride (TG), total cholesterol (TC), LDL, and HDL and homeostasis model of assessment index (HOMA-IR) at the baseline and after 8 weeks of intervention.
2.3. Blood Sampling and Laboratory Measurements. Venous blood samples $(10 \mathrm{ml})$ were drawn by venipuncture after 12-hour fasting at baseline and the end of the study. The blood samples were centrifuged for 10 minutes at $3000 \mathrm{rpm}$, and serum aliquoted into separate microtubes and were stored in $-80^{\circ} \mathrm{C}$ until biochemical analyses.

Serum levels of glucose and total cholesterol, LDL, HDL, and triglyceride were measured by an autoanalyzer instrument (BT 1500, Biotecnica Instruments, Italy), using commercial kits (Pars Azmoon, Co., Iran). The intra- and interassay coefficients of variations $(\mathrm{CV})$ for glucose, TC, HDL, LDL, and TG were $4.7 \%$ and $5.5 \%, 1.6 \%$ and $1.1 \%$, $0.8 \%$ and $1.8 \%, 0.6 \%$ and $1.2 \%$, and $1.53 \%$ and $1.6 \%$, respectively. Serum insulin was measured by using an enzymelinked immunosorbent assay (ELISA) kit (Monobind, Uppsala, US). The intra- and interassay CVs for insulin were 5.6 and $4.9 \%$, respectively. Insulin resistance was determined by calculating the HOMA-IR using the following formula: fasting glucose concentration $(\mathrm{mg} / \mathrm{dl}) \times$ fasting insulin level $(\mathrm{mU} / \mathrm{l}) / 405$ [40]. The serum level of ox-LDL was measured by using an ELISA kit (Shanghai Crystal Day Biotech Co., Ltd., China). CVs in intra- and interassay were 8 and 10\%, respectively. Lp-PLA2 mass was measured by using an ELISA kit (diaDexus, Inc. South San Francisco, CA, USA). The intra- and interassay CVs were $5.5 \%$ and $7.5 \%$, respectively.

2.4. Lp-PLA2 Distribution Determination. To determine the Lp-PLA2 distribution across HDL and apoB-containing lipoproteins, after precipitating the apoB lipoproteins from serum, HDL-Lp-PLA2 mass was measured using a LpPLA2 enzyme-linked immunosorbent assay (ELISA) kit (Changhai, Cristal Day Biotech Co., Ltd., China) on the obtained supernatant. The reagent containing $0.55 \mathrm{mM}$ phosphotungstic acid and $25 \mathrm{mM} \mathrm{MgCl} 2$ (Sigma-Aldrich, ST. Louis, MO) was used to precipitate the apoB lipoproteins. 200 microliters of serum sample was mixed with 500 microliters of precipitation reagent and incubated for five minutes at room temperature and centrifuged at $1500 \mathrm{~g}$ for ten minutes [41]. 200 microliters of apoB-depleted supernatant that contains only HDL were carefully separated without disrupting the precipitate and assayed for HDL-Lp-PLA2 mass.

Finally, apoB-Lp-PLA2 calculated by total Lp-PLA2 mass minus HDL-Lp-PLA2 [42]. The relative distribution of LpPLA2 was expressed as the percent of HDL-Lp-PLA2 [(HDL-Lp-PLA2/total Lp-PLA2 mass)*100] and the percent of apoB-associated Lp-PLA2 [(apoB-associated Lp-PLA2/total Lp-PLA2 mass)*100].

2.5. Anthropometric Assessment. Body weight was measured by a calibrated scale with $0.1 \mathrm{~kg}$ accuracy (Seca, Hamburg, Germany) with barefoot and minimal clothes. Height was measured by a wall-mounted tape, barefoot and at a straight standing posture with 0.5 -centimeter accuracy. BMI was calculated by dividing weight in kilograms by height in meters squared.

2.6. Assessment of Dietary Intake. To assess participant's food intake, we collected a $24 \mathrm{~h}$ food recall at the baseline and end 
of the study. The nutrition professional asked the patients to recall their intake by using a specific set of questions to gain as much detailed information as possible. To take the best result, for better estimating the portion size of the food and drinks, we used the Household Measures and Food Model booklet. Dietary intake was analyzed in terms of energy and macro- and micronutrients (with a focus on micronutrients with antioxidant activity) intake by Nutritionist version 4 that was modified for Iranian foods.

2.7. Assessment of Physical Activity Levels. To evaluate the physical activity levels, the Iranian version of the short form of International Physical Activity Questionnaires (IPAQ) was used at the baseline and the end of the study [43, 44]. The short form of IPAQ includes seven questions that explore walking and moderate-intensity and vigorous-intensity activity during the past seven days. Frequency (measured in days per week) and duration (time per day) are collected separately for each specific type of activity. To analyze the activities, MET-minute (metabolic equivalents per minute) score were used. The MET-minute score was computed by multiplying the MET score by the minutes performed and used categorical score.

The patients with at least 3000 met/minute score obtained from the combination of all the activities were considered category 3 (high) and with at least $600 \mathrm{met} / \mathrm{minute}$ score were considered category 2 (moderate), and patients not included in category 2 or 3 were considered category 1 (low) [45].

2.8. Statistical Analysis. The obtained data were analyzed with SPSS, version 21 (SPSS Inc. Chicago, IL, USA). An Intention-To-Treat (ITT) approach was used for data analysis. In this approach, all the enrolled participants who take $\geq$ one capsule were included. The multiple imputation method was used for predicting the missing values at the end of the study. In this method, we used a linear regression model to predict variables at the end of the study, by using values of the baseline and study group as the predictor. Results were expressed as mean \pm standard deviation (SD) and percentage for quantitative and qualitative variables, respectively. The Kolmogorov-Smirnov test detected the normality of variables, and if the distribution of a variable violated from the normal distribution, an appropriate transformation was performed. At the baseline, quantitative variables were analyzed by independent sample $t$-test and qualitative variables by chisquare test. A paired $t$-test was used to compare the mean variables before and after the intervention in each group, and two-way repeated measure ANOVA was used to identify the effect of the intervention on variables. To examine the correlation among variables and their changes, a Pearson correlation analysis was used. A $P$ value of less than 0.05 was considered statistically significant.

\section{Result}

Seventy diabetic patients were enrolled in the study and randomly allocated to the ALA group $(n=35)$ and the placebo group $(n=35)$. Three patients dropped out from the study, one from the ALA group (because of reporting the gastrointestinal complication) and two from the placebo group (because of unwillingness to continue). The study flow chart is depicted in Figure 1. After counting the returned capsules among those who completed the study, it was revealed the patients' compliance was high; about $98 \%$ of capsules in the ALA group and 96 percent in the placebo group were consumed. Since we applied the ITT approach, all the 70 participants were entered into the statistical analysis.

3.1. Baseline Characteristics and Dietary Intake. As depicted in Table 1, at the baseline, there were no substantial differences between the ALA and the placebo group in terms of patients' main characteristics. As expected, no significant differences were found in the BMI and physical activity level at the baseline and end of the study within and between the study groups. Dietary intakes are presented in Table 2. Energy and macro- and micronutrients intake were statistically similar between and within the two groups at the baseline and the end of the study.

3.2. Effect of ALA Supplementation on Lipid Profiles and Glycemic Indices. Lipid profiles (TC, LDL, HDL, and TG) and glycemic indices (glucose, insulin, and HOMA-IR) of participants at the baseline and after eight weeks of intervention are depicted in Table 3. There were no significant differences in terms of HOMA-IR, fasting serum glucose, insulin, TC, LDL, and HDL before and after the intervention in both groups. At the baseline of the study, no significant difference in serum TG was observed between the two groups. At the end of the study, TG reduced significantly in the ALA group $(P<0.001)$ and the significant time to group interaction effect was observed $(P=0.03)$.

3.3. Effect of ALA Supplementation on ox-LDL and APO A1. At the baseline of the study, ox-LDL and APO A1 levels had no significant differences between the two groups. After supplementation with ALA, a significant reduction in oxLDL $(P<0.001)$ and significant time to group interaction effect were observed $(P<0.001)$. There were no significant changes after intervention in APO A1 in both groups.

3.4. Effect of ALA Supplementation on Total Lp-PLA2 Mass and Its Distribution. The changes in total Lp-PLA2 mass, HDL-Lp-PLA2, apoB-associated Lp-PLA2, and percent of HDL-Lp-PLA2 across the time are presented in Figure 2. At the baseline, the two groups were statistically similar in terms of these variables. ALA supplementation reduced total Lp-PLA2 mass $(P=0.001)$ mainly by decreasing the apoB-associated Lp-PLA2 $(P=0.001)$ not HDL-Lp-PLA2 $(P=0.25)$. The time to group interaction effect was significant with regard to total Lp-PLA2 mass $(P=0.019)$ and apoB-associated Lp-PLA2 $(P=0.01)$. HDL-Lp-PLA2 had a nonsignificant reduction in both groups; also, time to group interaction effect was not significant.

As shown in Figure 3, however, the percent of HDL-LpPLA2 increased nonsignificantly in the ALA group and the time to group interaction effect was significant $(P=0.03)$. It should be noted that, although the increase in the percent of HDL-Lp-PLA2 in the ALA group was not statistically 


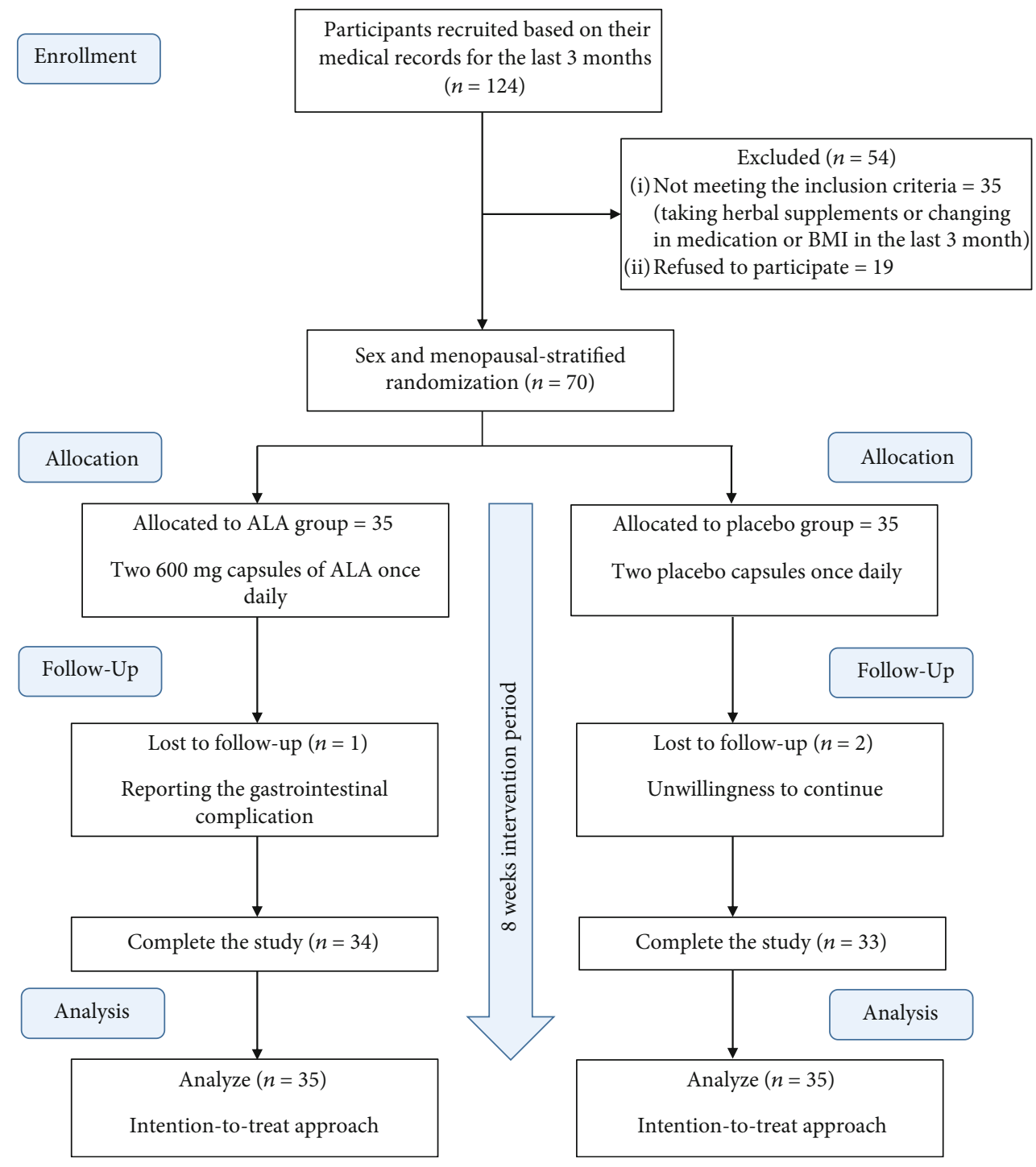

FIgURE 1: Study flow chart.

significant, it was very close to the significant level given the $P$ value $(P=0.051)$. Therefore, in the ALA group, the relative distribution of Lp-PLA2 improved by increasing the percent of HDL-Lp-PLA2 and a decrease in the percent of apoB-associated Lp-PLA2 compared to the placebo group.

3.5. Correlation Analysis. To assess the correlation among outcomes, the Pearson correlation test was performed. At the baseline of the study, we found a positive correlation between total Lp-PLA2 mass with TC $(r=0.355, P=0.003)$, LDL $(r=0.279, P=0.019)$, and ox-LDL $(r=0.762, P<$ $0.001)$. In addition, apoB-associated Lp-PLA2 was positively correlated with TC $(r=0.361, P=0.002)$, LDL $(r=0.292$, $P=0.014)$, and ox-LDL $(r=0.756, P<0.001)$ at the baseline of the study. As shown in Figure 4, there was a positive correlation among the changes of ox-LDL with changes of total Lp-PLA2 mass $(r=0.696, P<0.001)$ and apoB-associated Lp-PLA2 $(r=0.651, P<0.001)$ after supplementation with ALA. Furthermore, in the ALA group, the changes of percent of HDL-Lp-PLA2 were positively correlated with changes of
APO A1 $(r=0.335, P=0.049)$ but were negatively correlated with changes of TG $(r=-0.348, P=0.04)$.

3.6. Safety. According to a review article, supplementation with $600-2100 \mathrm{mg} /$ day ALA has not associated with serious adverse health effects [32]. However, in some studies, side effects such as itching, urticaria, fever, and epigastria soreness (heart burning) have been reported in subjects taking the dosage of $1200 \mathrm{mg} /$ day [46]. In our study, after supplementing with $1200 \mathrm{mg} /$ day ALA for eight weeks, the participants had no complaints of side effects except one person who complained of heart burning after five weeks of supplementation. This problem disappeared after discontinuing the ALA supplement taking.

\section{Discussion}

The results of this double-blind randomized, parallel, placebo-controlled clinical trial revealed that supplementation with $1200 \mathrm{mg}$ ALA for 8 weeks led to a significant 
TABLE 1: Baseline characteristics of participants.

\begin{tabular}{|c|c|c|c|}
\hline Variable & ALA group $(n=35)$ & Placebo group $(n=35)$ & $P$ value \\
\hline Age $(\text { year })^{\mathrm{a}}$ & $52.66(4.81)$ & $53.34(4.45)$ & 0.53 \\
\hline Female/male ${ }^{\mathrm{b}}$ & $20(57.1) / 15(42.9)$ & $19(54.3) / 16(45.7)$ & 0.81 \\
\hline Menopause ${ }^{b}$ & $13(37.1)$ & $14(40)$ & 0.81 \\
\hline Duration of diabetes (year) ${ }^{\mathrm{a}}$ & $3.65(1.67)$ & $3.57(1.83)$ & 0.83 \\
\hline $\mathrm{HbA} 1 \mathrm{C}^{\mathrm{a}}$ & $6.6(0.38)$ & $6.7(0.21)$ & 0.17 \\
\hline $\mathrm{BMI}^{\mathrm{a}}$ & $25.4(1.25)$ & $24.93(1.26)$ & 0.11 \\
\hline \multicolumn{4}{|l|}{ Medication $^{\mathrm{b}}$} \\
\hline Metformin & $32(91.4)$ & $31(88.6)$ & 0.69 \\
\hline Sulfonylurea & $8(22.9)$ & $10(28.6)$ & 0.58 \\
\hline $\mathrm{ARB}$ & $28(80)$ & $27(77.1)$ & 0.77 \\
\hline ACEI & $4(11.4)$ & $5(14.3)$ & 0.72 \\
\hline Statins & $29(82.9)$ & $30(85.7)$ & 0.74 \\
\hline Aspirin & $29(82.9)$ & $27(77.1)$ & 0.55 \\
\hline \multicolumn{4}{|l|}{ Physical activity level $^{\mathrm{b}}$} \\
\hline Low & $20(57.1)$ & $21(60)$ & \multirow{2}{*}{0.80} \\
\hline Moderate & $15(42.9)$ & $14(40)$ & \\
\hline
\end{tabular}

${ }^{\mathrm{a}}$ Presented as mean (SD), $P$ values are resulted from independent sample t-test. ${ }^{\mathrm{b}}$ Presented as number (\%), $P$ values are resulted from a chi-square test. Low physical activity: score less than 600 MET-minutes/week and moderate physical activity: score between 600 and 3000 MET-minutes/week.

reduction in total Lp-PLA2 mass, ox-LDL, and TG level and improved the distribution of Lp-PLA2 between HDL and apoB lipoproteins. Nevertheless, there was no significant effect on glycemic indices (such as FBS, insulin, and HOMA-IR), TC, HDL, LDL, and Apo A1 in type 2 diabetic patients.

Since at the baseline of the study, there were no substantial differences in medication distribution between the groups, and making any changes in medications three months before and during the study was one of the exclusion criteria, and in addition, there were no changes in the dietary intake and physical activity levels of participants during the study; therefore, our results can be attributed to ALA.

To our knowledge, it is the first clinical trial that examines the effect of ALA on Lp-PLA2 mass and its distribution among lipoproteins in type 2 diabetes patients.

ALA is synthesized de novo from medium-chain fatty acid, called octanoic acid, in small quantity in the body. ALA is found in food sources, such as red meat, the liver, the heart, and the kidney, and to a lesser degree, in spinach, broccoli, tomatoes, Brussel sprouts, potatoes, garden peas, and rice bran. There is evidence that food intake does not meet the appreciable amount of ALA; rather, oral dietary supplements are considered a primary source of ALA. The amount of ALA available in dietary supplements is about up to 1000 times more than the amount that could be obtained from diet. It has been shown that bioavailability of ALA from oral supplements is about $20-40 \%$, while the efficiency of ALA absorption from the food source due to competition with other nutrients for carrier proteins is less. Therefore, it is recommended that ALA be taken $30 \mathrm{~min}$ before or 2 hours after a meal [27, 47].

Lp-PLA2, formerly named platelet-activating factor acetylhydrolase (PAF-AH), is a novel specific biomarker for vascular inflammation and useful tool for diagnosing and cardiovascular disease risk assessment in diabetic and general population. This enzyme may play a biological role in the development and progression of atherosclerosis [17, 48]. Lp-PLA2 catalyzes the hydrolysis of ox-LDL in the arterial wall and produces two inflammatory mediators, lysophosphatidylcholine and oxidized fatty acids [10, 19, 49]. Therefore, it appears that Lp-PLA2 may mediate the atherogenic effect of ox-LDL. According to the in vitro studies, inhibition of Lp-PLA2 diminished the arterial lesions evoked by ox-LDL $[49,50]$. However, there is a controversy in the causal role of Lp-PLA2 in CHD. Some studies have shown that Lp-PLA2 was associated with CHD independent of conventional risk factors $[13,51,52]$. While in some GWAS, a variant in the PLA2G7 gene (V279F) that encodes the Lp-PLA2 with lower activity was not associated with cardiovascular risk markers, coronary atheroma, or CHD $[11,12]$.

In this study, $1200 \mathrm{mg}$ ALA after eight weeks significantly reduced total Lp-PLA2 mass and ox-LDL levels compared with the placebo. Furthermore, we found a positive correlation between the reduction in ox-LDL level and total LpPLA2 mass in the ALA group. Such a positive correlation has also been observed in other clinical studies [53-55].

Ox-LDL is involved in the incidence and progression of atherosclerosis and diabetic complications. It has been reported that in diabetic patients, ox-LDL levels rise as the disease progresses, regardless of serum LDL levels [56]. ALA is a potent radical scavenger and protects against ROS-induced lipid oxidative damage [24]. In in vitro studies, ALA prevented the oxidation of LDL induced via oxidizing metals, such as $\mathrm{Cu}^{2+}$, by metal chelating activity and sparing vitamin $\mathrm{E}$ as the main antioxidant carried on LDL [36, 57]. In consistent with our study, supplementation with $600 \mathrm{mg}$ ALA intravenously once daily for two weeks significantly decreased the plasma ox-LDL level in obese-impaired glucose tolerance patients [37]. 
TABLE 2: Dietary intake of participants at the baseline and after 8 weeks of intervention.

\begin{tabular}{|c|c|c|c|c|c|}
\hline $\begin{array}{l}\text { Variables } \\
\end{array}$ & & ALA group & Placebo group & $P$ value* & $P$ value $^{* *}$ \\
\hline \multirow{2}{*}{ Energy (kcal/day) } & Before & $2227.57(363.28)$ & $2269.74(356.95)$ & \multirow{2}{*}{0.62} & \multirow{2}{*}{0.62} \\
\hline & After & $2176.22(282.93)$ & $2240.28(345.06)$ & & \\
\hline \multirow{2}{*}{ Carbohydrate (g/day) } & Before & $302.61(52.31)$ & $307.73(49.54)$ & \multirow{2}{*}{0.67} & \multirow{2}{*}{0.89} \\
\hline & After & $303.17(50.76)$ & $309.06(47.16)$ & & \\
\hline \multirow{2}{*}{ Carbohydrate (\%) } & Before & $54.28(2.46)$ & $54.24(2.40)$ & \multirow{2}{*}{0.94} & \multirow{2}{*}{0.86} \\
\hline & After & $55.60(4.63)$ & $55.37(4.80)$ & & \\
\hline \multirow{2}{*}{ Protein (g/day) } & Before & $61.20(9.28)$ & $60.31(10.48)$ & \multirow{2}{*}{0.70} & \multirow{2}{*}{0.33} \\
\hline & After & $60.02(7.49)$ & $60.66(9.87)$ & & \\
\hline \multirow{2}{*}{ Protein (\%) } & Before & $11.03(0.74)$ & $10.64(0.94)$ & \multirow{2}{*}{0.06} & \multirow{2}{*}{0.43} \\
\hline & After & $11.04(0.41)$ & $10.82(0.54)$ & & \\
\hline \multirow{2}{*}{ Fat (g/day) } & Before & $85.80(14.91)$ & $88.61(15.99)$ & \multirow{2}{*}{0.45} & \multirow{2}{*}{0.72} \\
\hline & After & $80.38(14.08)$ & $84.59(19.81)$ & & \\
\hline \multirow{2}{*}{ Fat $(\%)$} & Before & $34.67(2.37)$ & $35.10(2.36)$ & \multirow{2}{*}{0.45} & \multirow{2}{*}{0.98} \\
\hline & After & $33.34(4.61)$ & $33.79(4.90)$ & & \\
\hline \multirow{2}{*}{ Vitamin A ( $\mu \mathrm{g} /$ day) } & Before & $646.30(132.88)$ & $618.13(92.49)$ & \multirow{2}{*}{0.30} & \multirow{2}{*}{0.66} \\
\hline & After & $647.51(126.12)$ & $622.71(100.52)$ & & \\
\hline \multirow{2}{*}{ Vitamin E (mg/day) } & Before & $18.63(4.12)$ & $19.23(4.20)$ & \multirow{2}{*}{0.54} & \multirow{2}{*}{0.34} \\
\hline & After & $18.20(3.82)$ & $18.15(4.51)$ & & \\
\hline \multirow{2}{*}{ Vitamin C (mg/day) } & Before & $79.20(10.50)$ & $83.05(13.06)$ & \multirow{2}{*}{0.17} & \multirow{2}{*}{0.43} \\
\hline & After & $81.36(10.66)$ & $86.42(15.18)$ & & \\
\hline \multirow{2}{*}{ Riboflavin (mg/day) } & Before & $1.60(0.43)$ & $1.63(0.49)$ & \multirow{2}{*}{0.74} & \\
\hline & After & $1.59(0.40)$ & $1.62(0.52)$ & & 0.86 \\
\hline & Before & $9.68(1.93)$ & $9.95(2.20)$ & & \\
\hline Zinc (mg/day) & After & $9.94(1.94)$ & $10.28(2.23)$ & 0.59 & 0.84 \\
\hline & Before & $67.06(11.59)$ & $64.09(15.16)$ & & \\
\hline Selenium ( $\mu \mathrm{g} /$ day) & After & $67.83(11.91)$ & $65.17(15.80)$ & 0.36 & 0.75 \\
\hline & Before & $8.89(2.66)$ & $9.67(3.50)$ & & \\
\hline Iron (mg/day) & After & $9.14(2.25)$ & $10.06(2.65)$ & 0.29 & 0.75 \\
\hline
\end{tabular}

The results are described as mean (SD). ${ }^{*}$ according to independent $t$-test at baseline; ${ }^{* *}$ time to group interaction according to two-way repeated measure ANOVA.

The underlying mechanisms of the effects of ALA on LpPLA2 mass are unclear. It has been shown that ox-LDL is a potent stimulator of Lp-PLA2 expression by activating the P38 mitogen activating protein kinase (MAPK) pathway [49]. Therefore, the beneficial effects of ALA supplementation against Lp-PLA2 that are observed in our study might result from its reductive effects on ox-LDL serum levels. On the other hand, according to some in vitro studies, ALA attenuates P38 MAPK activation [58-60]. Then, we can consider the possibility that ALA indirectly downregulates Lp-PLA2 gene expression by suppressing the P38 MAPK activation. Furthermore, in an in vitro study, ALA suppressed Lp-PLA2 gene expression in THP-1 derived macrophages after stimulating these cells by $\mathrm{H}_{2} \mathrm{O}_{2}$ in order to induce the Lp-PLA2 gene expression [38].

The vast majority of plasma Lp-PLA2 is bound to apoB-containing lipoproteins, including LDL, VLDL, and IDL, and a smaller amount is associated with HDL; hence, apoB-associated Lp-PLA2 is the primary determinant of the total Lp-PLA2 level. It has been illustrated that HDL-LpPLA2 has anti-inflammatory and antiatherogenic properties, whereas apoB-associated Lp-PLA2 has proinflammatory activity [6]. A three-year follow-up prospective study in patients with stable CAD reported that higher HDL-LpPLA2 levels and a lower ratio of Lp-PLA2 mass to HDLLp-PLA2 are related to a lower risk for cardiac mortality, independent of other conventional cardiovascular risk factors [61]. According to these considerations, it has been suggested that the relative distribution of Lp-PLA2 may be a better cardiovascular risk marker rather than the total LpPLA2 level [23].

Darapladib, a selective Lp-PLA2 inhibitor, in both clinical trials and animal studies reduced the necrotic core volume and arterial lesions but did not reduce the rate of cardiovascular death, myocardial infarction, or stroke in patients with stable CHD [62-64]. It should be noted that all studies on darapladib have been focus on total Lp-PLA2 activity and there is no information on its effect on HDLLp-PLA2 and Lp-PLA2 distribution [6]. Since in an in vitro study inhibition of HDL-Lp-PLA2 by darapladib diminished the antioxidative and vasoprotective properties of HDL [65], it is likely that failure to reduce in CVD events be due to 
TABLE 3: BMI and biochemical parameters of participants at the baseline and after 8 weeks of intervention.

\begin{tabular}{|c|c|c|c|c|c|}
\hline Variables & & Before & After & $P$ value* & $P$ value ${ }^{* *}$ \\
\hline \multirow{2}{*}{ BMI $\left(\mathrm{kg} / \mathrm{m}^{2}\right)$} & ALA group & $25.41(1.25)$ & $25.32(1.27)$ & 0.13 & \multirow{2}{*}{0.16} \\
\hline & Placebo group & $24.93(1.26)$ & $20 \times 4.99(1.15)$ & 0.52 & \\
\hline \multirow{2}{*}{ APO Al (mg/dl) } & ALA group & $123.56(18.99)$ & $128.34(19.42)$ & 0.06 & \multirow{2}{*}{0.09} \\
\hline & Placebo group & $126.17(22.13)$ & $123.00(22.62)$ & 0.42 & \\
\hline \multirow{2}{*}{ Ox-LDL (ng/l) } & ALA group & $42.82(1.44)$ & $32.14(1.46)$ & 0.00 & \multirow{2}{*}{0.00} \\
\hline & Placebo group & $35.89(1.61)$ & 34.43 (1.71) & 0.19 & \\
\hline \multirow{2}{*}{$\mathrm{TC}(\mathrm{mg} / \mathrm{dl})$} & ALA group & $159.91(24.22)$ & $151.74(21.92)$ & 0.15 & \multirow{2}{*}{0.30} \\
\hline & Placebo group & $153.63(21.00)$ & $151.69(17.80)$ & 0.43 & \\
\hline \multirow{2}{*}{ HDL (mg/dl) } & ALA group & $47.43(5.87)$ & $45.74(7.64)$ & 0.25 & \multirow{2}{*}{0.98} \\
\hline & Placebo group & $45.37(4.88)$ & $43.71(6.87)$ & 0.26 & \\
\hline \multirow{2}{*}{$\mathrm{LDL}$ (mg/dl) } & ALA group & $78.18(22.74)$ & $76.01(23.37)$ & 0.66 & \multirow{2}{*}{0.72} \\
\hline & Placebo group & $71.94(18.70)$ & $71.66(13.38)$ & 0.89 & \\
\hline \multirow{2}{*}{$\mathrm{TG}(\mathrm{mg} / \mathrm{dl})$} & ALA group & $171.26(31.86)$ & $149.03(39.23)$ & 0.00 & \multirow{2}{*}{0.03} \\
\hline & Placebo group & $181.14(50.42)$ & $180.11(52.18)$ & 0.89 & \\
\hline \multirow{2}{*}{ FBS (mg/dl) } & ALA group & $134.03(11.56)$ & $127.74(15.26)$ & 0.07 & \multirow{2}{*}{0.06} \\
\hline & Placebo group & $131.51(14.92)$ & $133.51(11.71)$ & 0.48 & \\
\hline \multirow{2}{*}{ Insulin (mU/l) } & ALA group & $10.83(1.53)$ & $10.74(1.55)$ & 0.54 & \multirow{2}{*}{0.38} \\
\hline & Placebo group & $10.94(1.63)$ & $11.12(1.14)$ & 0.51 & \\
\hline \multirow{2}{*}{ HOMA-IR } & ALA group & $3.58(0.58)$ & $3.38(0.62)$ & 0.07 & \multirow{2}{*}{0.058} \\
\hline & Placebo group & $3.55(0.66)$ & $3.67(0.53)$ & 0.34 & \\
\hline
\end{tabular}

The results are described as mean (SD). BMI: body mass index; Apo A1: apolipoprotein A1; ox-LDL: oxidized low-density lipoprotein; TC: total cholesterol; HDL: high-density lipoprotein cholesterol; LDL: low-density lipoprotein cholesterol; TG: triglyceride; FBS: fasting blood sugar; HOMA-IR: homeostasis model of assessment index. * according to paired t-test; ${ }^{* *}$ time to group interaction according to two-way repeated measure ANOVA.

inhibition of HDL-Lp-PLA2 by darapladib and its effect on the Lp-PLA2 distribution.

In T2D patients, the impaired Lp-PLA2 distribution, which is characterized by lower HDL-Lp-PLA2 and higher apoB-associated Lp-PLA2, has been reported [17]. Passaro et al. showed that the incidence of type 2 diabetes might coincide with a shift of Lp-PLA2 towards the atherogenic lipoproteins particles [21].

As far as we know, this is the first study to investigate the effect of an oral nutritional supplement intervention on LpPLA2 distribution among lipoproteins. In our study, the decrease in total Lp-PLA2 after supplementation with ALA was associated with a significant decline in apoB-associated Lp-PLA2 and a nonsignificant reduction in HDL-Lp-PLA2, so resulting in an increase in the percent of HDL-Lp-PLA2 and a decrease in the percent of apoB-associated Lp-PLA2. These findings show that ALA improved the distribution of Lp-PLA2 among lipoproteins. In addition, we observed that in the ALA group, changes in the percent of HDL-Lp-PLA2 had a positive correlation with changes of APO A1 and a negative correlation with changes of TG.

It is unclear how ALA significantly increased the percent of HDL-Lp-PLA2 and improved the Lp-PLA2 distribution. Considering the reduction in the TG level and the negative correlation between TG changes and the percent of HDLLp-PLA2 after supplementation with ALA, one possible explanation is that since ALA may enhance the lipolysis of triglyceride-rich apoB-containing lipoproteins by activating the lipoprotein lipase (LPL) [37], probably, the Lp-PLA2s that bound to these lipolyzed lipoproteins have transferred to the HDL lipoproteins. Apparently, fibrates improve LpPLA2 distribution through such a mechanism [66].

APO A1 is the main structural protein of HDL and is a mediator in reverse cholesterol transport. In experimental and clinical studies, the upregulation of APO A1 was associated with a reduction in atherosclerosis [67]. APO AI overexpression resulted in a substantial increase in HDLLp-PLA2 in an animal model study [68]; in addition, the positive correlation between APO A1 and HDL-Lp-PLA2 has been reported $[6,69]$. On the other hand, ALA may increase APO A1 synthesis in the liver [37]. So based on these evidences, we assumed that ALA possibly increase HDLLp-PLA2 and improve Lp-PLA2 distribution via APO A1 increase. However, in our study, the increase in APO A1 was not significant; this increase was positively correlated with the increase in the percent of HDL-Lp-PLA2.

According to in vitro studies, ALA stimulates translocation of glucose transporter 4 (GLUT4) to the membrane of fat and skeletal muscle cells, raises the activity of component of insulin signaling, and induces the expression of PPAR- $1 \alpha$ and PPAR-1 $\gamma$. Therefore, it may improve glucose hemostasis in diabetic patients [70, 71].

In our study, ALA supplementation could not improve the serum levels of glucose, insulin, and HOMA-IR. In accordance with our findings, de Oliviera et al. reported that treatment with $600 \mathrm{mg}$ ALA for 20 weeks had no significant effect on serum levels of glucose, insulin, and HOMA-IR [72]. Likewise, Mendoza et al. found no significant reduction in serum 


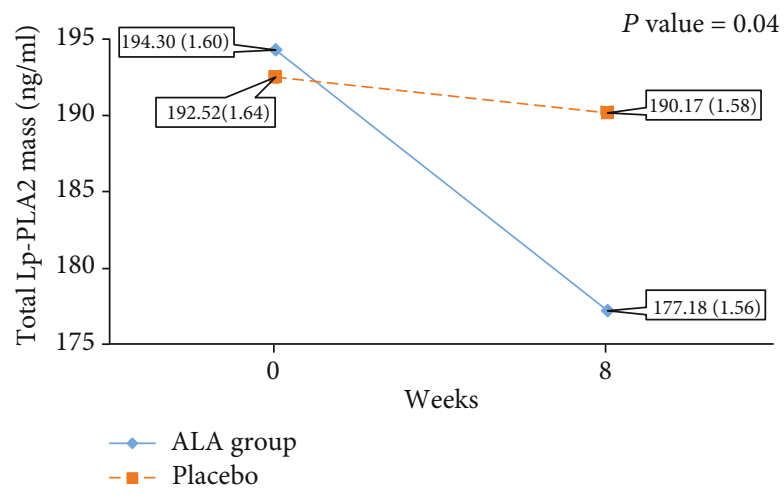

(a)

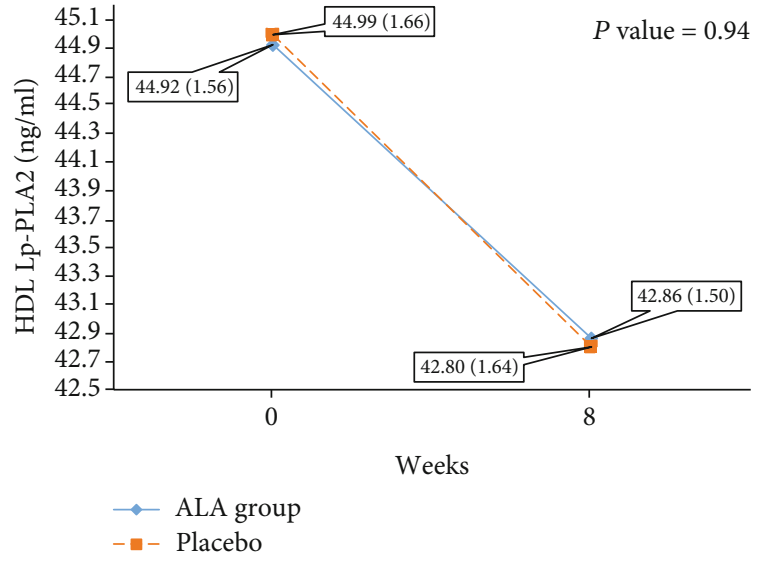

(b)

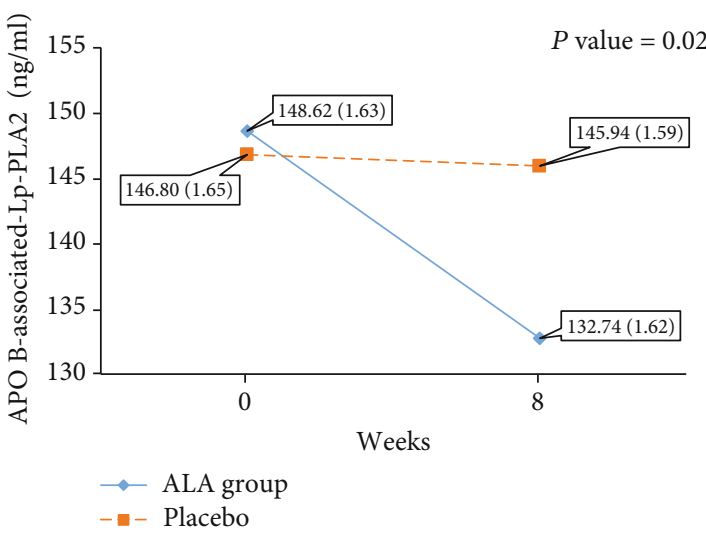

(c)

Figure 2: The changes in total Lp-PLA2 mass (a), HDL-Lp-PLA2 (b), and apoB-associated Lp-PLA2 (c) at the baseline and after 8 weeks of intervention. $P$ value $<0.05$ indicates a significant difference between groups based on the interaction effect shown by the two-way repeated measured ANOVA.

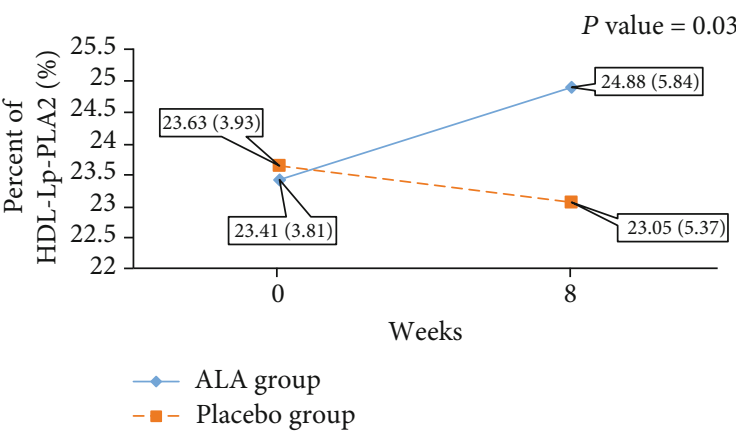

(a)

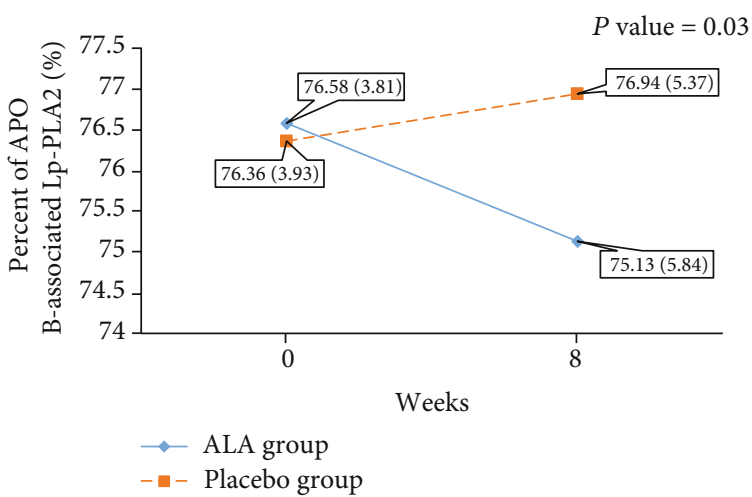

(b)

Figure 3: The changes in the percent of HDL-Lp-PLA2 (a) and the percent of apoB-associated Lp-PLA2 (b) at the baseline and after 8 weeks of intervention. Percent of HDL-Lp-PLA2: (HDL-Lp-PLA2/Lp-PLA2 mass) * 100; percent of apoB-associated Lp-PLA2: (apoB-associated Lp-PLA2/Lp-PLA2 mass) $* 100 . P$ value $<0.05$ indicates a significant difference between groups based on the interaction effect shown by the two-way repeated measured ANOVA.

glucose after supplementation with $600 \mathrm{mg}$ ALA for six months [73]. In contrast, in one clinical study, after eight weeks of supplementation with $300 \mathrm{mg}$ ALA in type 2 diabetic patients, an improvement in serum levels of glucose and HOMA-IR was reported [74]. Furthermore, supplemen- tation with a food supplement containing $600 \mathrm{mg}$ ALA after three months has shown a significant reduction in the serum level of glucose in patients with type 2 diabetes [31]. A possible explanation for our results is that all of our study population, similar to Oliveria's study, were well-controlled diabetic 


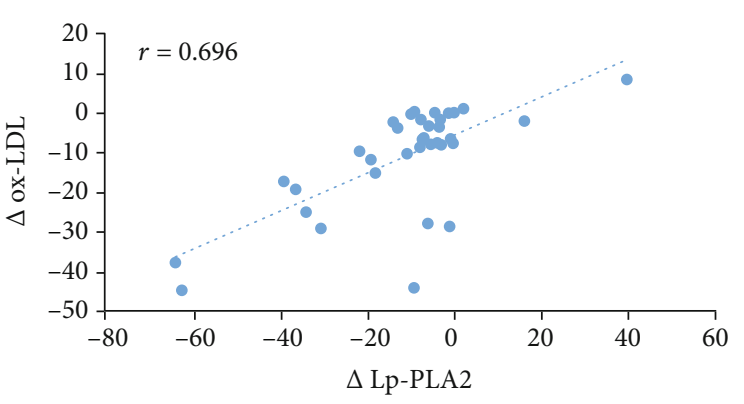

(a)

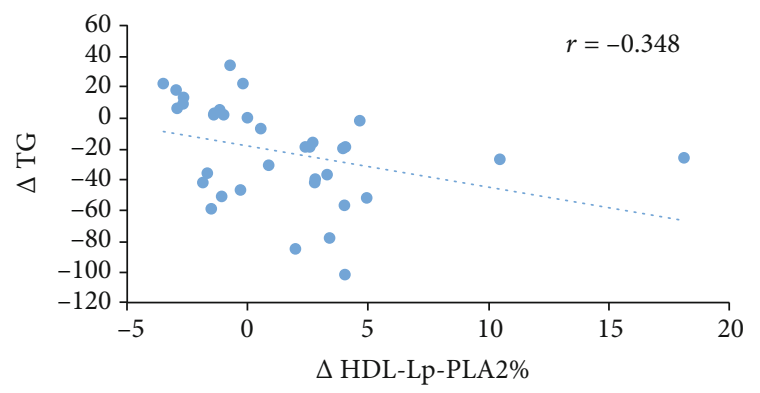

(c)

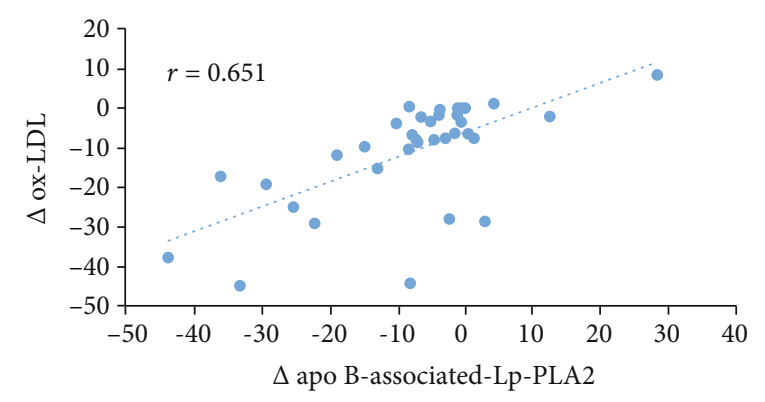

(b)

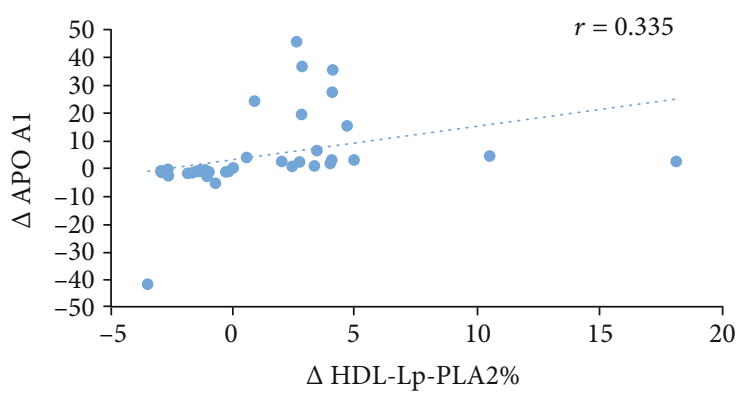

(d)

FIGURE 4: Correlation between changes of ox- LDL and total Lp-PLA2 mass (a), changes of ox-LDL and apoB-associated Lp-PLA2 mass (b), changes of TG and percent of HDL-Lp-PLA2 (c), and changes of APO A1 and percent of HDL-Lp-PLA2 (d) after supplementation with ALA.

patients because one of our inclusion criteria was $\mathrm{HbA} 1 \mathrm{C} \leq$ $7 \%[72]$.

Little is known about the lipid-lowering effect of ALA in diabetic patients, and there are conflicting reports in this regard. In our study, after supplementation with $1200 \mathrm{mg}$ ALA for eight weeks, we have found a nonsignificant effect on serum levels of TC, LDL, and HDL but a significant reduction in the TG level. Zhang et al. reported a significant reduction in serum levels of TC, LDL, and TG and a significant increase in HDL levels after intravenous administration of $600 \mathrm{mg}$ ALA once daily for two weeks in obese-IGT patients [37]. In a study by Mendoza et al., after treatment with $600 \mathrm{mg}$ ALA for six months in type 2 diabetic patients, a significant reduction in TC and an increase in HDL levels were reported; however, any significant change in TG levels was not observed [73]. In another study, supplementation with $600 \mathrm{mg}$ ALA for 20 weeks in diabetic patients led to a nonsignificant effect on serum levels of TC, LDL, HDL, and TG [72].

The potential mechanism of the ALA effect on the lipid profile is not well understood. ALA may lower TC and LDL by [1] downregulating the squalene monooxygenase gene [2], increasing the LPL [3], synthesizing the LDL receptors in the liver which causes enhancing cholesterol uptake and increasing APO A1 synthesize [4], and inhibiting the oxidation of LDL to ox-LDL which improves the hepatic LDL receptor recognition and plasma clearance [32, 37]. In addition, ALA may improve TG levels via downregulating glycerol-3-phosphate acyltransferase, a rate-limiting enzyme of TG biosynthesize; enhancing lipolysis of TG-rich lipoproteins by activating LPL; and inhibiting the acetyl-CoA carboxylase activity $[37,75]$. A meta-analysis study indicated that ALA supplementation resulted in a significant reduction in the serum level of TC, LDL, and TG but not on increasing the HDL level. According to this study, supplementation dosage, treatment duration, and baseline participants' BMI and lipid levels are determining factors that influence the lipidlowering potential of ALA. More effects have been observed in higher ALA dosage $(600 \mathrm{mg})$, treatment duration more than 12 weeks, and baseline BMI > 30 [75]. In our study, $82.9 \%$ of participants are taking statins and had normal serum levels of TC and LDL, so it appears they had less room for improvement. On the other hand, the participants mean baseline TG levels were higher than normal levels, and none of the participants were taking TG-lowering drugs such as fibrates. These can be a possible explanation for our results.

4.1. Limitations. One of the study limitations is that due to financial and time constraints, we could not investigate whether observed ALA positive effects are associated with the prevention of cardiovascular endpoints and atherosclerotic lesions determined either by ultrasonography or by angiographic techniques. Further, since the laboratory methods used to measure the blood ALA levels are complex and costly, we had not been able to evaluate patient compliance accurately. However, we compensated this limitation to some extent through frequent and regular telephone calls and counting the remaining capsules, so we observed a $98 \%$ compliance rate in these patients. Another limitation of our study was using $24 \mathrm{~h}$ recall instead of food record to assess the dietary intake due to the limited literacy skill of some participants to measure or judge the food portion size and probability of noncompliance with the food record submission. Also, unfortunately, because of the participants' commuting difficulties, it was not possible to have more visits to obtain $24 \mathrm{~h}$ recall during the study. 


\section{Conclusion}

In this double-blind, randomized, parallel, placebocontrolled clinical trial, supplementation with $1200 \mathrm{mg}$ ALA after eight weeks resulted in a substantial reduction in oxLDL and Lp-PLA2 mass and improvement in Lp-PLA2 distribution between HDL and apoB-containing lipoproteins in type 2 diabetic patients. These observed desired effects may be associated with the reduction in the risk of CVD in these patients. Further clinical trial studies should be conducted to evaluate the cardiovascular endpoints and cardioprotective effects of this supplement in the long term.

\section{Data Availability}

The data (database in Excel) used to support the findings of this study are available from the corresponding or first author upon request. (saeedhmdphd@hotmail.com, nbaziar@razi.tums.ac.ir)

\section{Conflicts of Interest}

The authors declare that they have no competing interests.

\section{Acknowledgments}

This study was supported by a research grant from Tehran University of Medical Sciences and Health Services. We are immensely grateful to participants for their collaboration. We would also like to show our gratitude to the staffs at the Diabetes Research Center, Endocrinology and Metabolism Research Center, Tehran University of Medical Sciences, especially to Mrs. Pourreza for relentless assistance in all sampling procedures.

\section{References}

[1] S. Schwartz and B. A. Kohl, "Type 2 diabetes mellitus and the cardiometabolic syndrome: impact of incretin-based therapies," Diabetes, Metabolic Syndrome and Obesity: Targets and Therapy, vol. 3, pp. 227-242, 2010.

[2] C. C. Low Wang, C. N. Hess, W. R. Hiatt, and A. B. Goldfine, "Clinical update: cardiovascular disease in diabetes mellitus: atherosclerotic cardiovascular disease and heart failure in type 2 diabetes mellitus - mechanisms, management, and clinical considerations," Circulation, vol. 133, no. 24, pp. 2459-2502, 2016.

[3] N. Katakami, "Mechanism of development of atherosclerosis and cardiovascular disease in diabetes mellitus," Journal of Atherosclerosis and Thrombosis, vol. 25, no. 1, pp. 27-39, 2018.

[4] D. Li, L. Zhao, J. Yu et al., "Lipoprotein-associated phospholipase A2 in coronary heart disease: review and meta-analysis," Clinica Chimica Acta, vol. 465, pp. 22-29, 2017.

[5] M. H. Davidson, M. A. Corson, M. J. Alberts et al., "Consensus panel recommendation for incorporating lipoprotein-associated phospholipase $\mathrm{A}_{2}$ testing into cardiovascular disease risk assessment guidelines," The American Journal of Cardiology, vol. 101, no. 12, pp. S51-S57, 2008.

[6] C. Tellis and A. Tselepis, "Pathophysiological role and clinical significance of lipoprotein-associated phospholipase A2
(Lp-PLA2) bound to LDL and HDL," Current Pharmaceutical Design, vol. 20, no. 40, pp. 6256-6269, 2014.

[7] I. J. Hatoum, F. B. Hu, J. J. Nelson, and E. B. Rimm, "Lipoprotein-associated phospholipase A2 activity and incident coronary heart disease among men and women with type 2 diabetes," Diabetes, vol. 59, no. 5, pp. 1239-1243, 2010.

[8] K. Kotani, "Plasma lipoprotein-associated phospholipase A2 levels correlated with the cardio-ankle vascular index in long-term type 2 diabetes mellitus patients," International Journal of Molecular Sciences, vol. 17, no. 5, p. 634, 2016.

[9] J. Seyfarth, T. Reinehr, A. Hoyer et al., "Lipoprotein-associated phospholipase A2 activity in obese adolescents with and without type 2 diabetes," Journal of Inherited Metabolic Disease, vol. 41, no. 1, pp. 73-79, 2018.

[10] K. J. Colley, R. L. Wolfert, and M. E. Cobble, "Lipoprotein associated phospholipase A2: role in atherosclerosis and utility as a biomarker for cardiovascular risk," The EPMA Journal, vol. 2, no. 1, pp. 27-38, 2011.

[11] J. P. Casas, E. Ninio, A. Panayiotou et al., "PLA2G7 genotype, lipoprotein-associated phospholipase A2 activity, and coronary heart disease risk in 10494 cases and 15624 controls of European ancestry,” vol. 121, no. 21, pp. 2284-2293, 2010.

[12] I. Y. Millwood, D. A. Bennett, R. G. Walters et al., "A phenome-wide association study of a lipoprotein-associated phospholipase A2 loss-of-function variant in 90000 Chinese adults," International Journal of Epidemiology, vol. 45, no. 5, pp. 1588-1599, 2016.

[13] Y. Jang, O. Y. Kim, S. J. Koh et al., “The Val279Phe variant of the lipoprotein-associated phospholipase A2 gene is associated with catalytic activities and cardiovascular disease in Korean men," The Journal of Clinical Endocrinology \& Metabolism, vol. 91, no. 9, pp. 3521-3527, 2006.

[14] T. L. Nelson, M. L. Biggs, J. R. Kizer et al., "Lipoprotein-associated phospholipase A2 (Lp-PLA2) and future risk of type 2 diabetes: results from the cardiovascular health study," The Journal of Clinical Endocrinology \& Metabolism, vol. 97, no. 5, pp. 1695-1701, 2012.

[15] A. De Stefano, L. Mannucci, F. Tamburi et al., "Lp-PLA2, a new biomarker of vascular disorders in metabolic diseases," International Journal of Immunopathology and Pharmacology, vol. 33, 2019.

[16] L. Jackisch, W. Kumsaiyai, J. D. Moore et al., "Differential expression of Lp-PLA2 in obesity and type 2 diabetes and the influence of lipids," Diabetologia, vol. 61, no. 5, pp. 11551166, 2018.

[17] H. A. Zhu, "Lp-PLA2, a novel potential biomarker predicting cardiovascular disease in type 2 diabetes mellitus," Medical \& Clinical Reviews, vol. 2, no. 2, 2016.

[18] S. Garg, S. Madhu, and S. Suneja, "Lipoprotein associated phospholipase A2 activity \& its correlation with oxidized LDL \& glycaemic status in early stages of type-2 diabetes mellitus," Indian Journal of Medical Research, vol. 141, no. 1, pp. 107-114, 2015.

[19] H. Zheng, D. J. Cui, X. J. Quan et al., "Lp-PLA2 silencing protects against ox-LDL-induced oxidative stress and cell apoptosis via Akt/mTOR signaling pathway in human THP1 macrophages," Biochemical and Biophysical Research Communications, vol. 477, no. 4, pp. 1017-1023, 2016.

[20] J. L. Sánchez-Quesada, I. Vinagre, E. De Juan-Franco et al., "Impact of the LDL subfraction phenotype on Lp-PLA2 
distribution, LDL modification and HDL composition in type 2 diabetes," Cardiovascular Diabetology, vol. 12, no. 1, p. 112, 2013.

[21] A. Passaro, G. B. Vigna, A. Romani et al., "Distribution of paraoxonase-1 (PON-1) and lipoprotein phospholipase A2 (Lp-PLA2) across lipoprotein subclasses in subjects with type 2 diabetes," Oxidative Medicine and Cellular Longevity, vol. 2018, Article ID 1752940, 10 pages, 2018.

[22] F. Huang, K. Wang, and J. Shen, "Lipoprotein-associated phospholipase A2: the story continues," Medicinal Research Reviews, vol. 40, no. 1, pp. 79-134, 2019.

[23] J. L. Sánchez-Quesada, I. Vinagre, E. de Juan-Franco et al., "Effect of improving glycemic control in patients with type 2 diabetes mellitus on low-density lipoprotein size, electronegative low-density lipoprotein and lipoprotein-associated phospholipase A2 distribution," The American Journal of Cardiology, vol. 110, no. 1, pp. 67-71, 2012.

[24] B. Skibska and A. Goraca, "The protective effect of lipoic acid on selected cardiovascular diseases caused by age-related oxidative stress," Oxidative Medicine and Cellular Longevity, vol. 2015, Article ID 313021, 11 pages, 2015.

[25] S. Salinthone, V. Yadav, D. N. Bourdette, and D. W. Carr, "Lipoic acid: a novel therapeutic approach for multiple sclerosis and other chronic inflammatory diseases of the CNS," Endocrine, Metabolic \& Immune Disorders - Drug Targets, vol. 8, no. 2, pp. 132-142, 2008.

[26] S. H. K. Ali and W. A. Raja, "The effect of antioxidants in acute amitriptyline poisoning," Toxicology Reports, vol. 6, pp. 380388, 2019.

[27] H. Moini, L. Packer, and N. E. Saris, "Antioxidant and prooxidant activities of alpha-lipoic acid and dihydrolipoic acid," Toxicology and Applied Pharmacology, vol. 182, no. 1, pp. 84-90, 2002.

[28] S. Vasdev, V. Gill, and P. Singal, "Modulation of oxidative stress-induced changes in hypertension and atherosclerosis by antioxidants," Experimental and Clinical Cardiology, vol. 11, no. 3, pp. 206-216, 2006.

[29] K. Jomova and M. Valko, "Health protective effects of carotenoids and their interactions with other biological antioxidants," European Journal of Medicinal Chemistry, vol. 70, pp. 102110, 2013.

[30] L. Holmquist, G. Stuchbury, K. Berbaum et al., "Lipoic acid as a novel treatment for Alzheimer's disease and related dementias," Pharmacology \& Therapeutics, vol. 113, no. 1, pp. 154-164, 2007.

[31] G. Derosa, A. D’Angelo, D. Romano, and P. Maffioli, “A clinical trial about a food supplement containing $\alpha$-Lipoic acid on oxidative stress markers in type 2 diabetic patients," International Journal of Molecular Sciences, vol. 17, no. 11, p. 1802, 2016.

[32] S. V. Harding, T. C. Rideout, and P. J. H. Jones, "Evidence for using alpha-lipoic acid in reducing lipoprotein and inflammatory related atherosclerotic risk," Journal of Dietary Supplements, vol. 9, no. 2, pp. 116-127, 2012.

[33] Z. Amom, Z. Zakaria, J. Mohamed et al., "Lipid lowering effect of antioxidant alpha-lipoic acid in experimental atherosclerosis," Journal of Clinical Biochemistry and Nutrition, vol. 43, no. 2, pp. 88-94, 2008.

[34] W.-R. Lee, A. Kim, K.-S. Kim et al., "Alpha-lipoic acid attenuates atherosclerotic lesions and inhibits proliferation of vascular smooth muscle cells through targeting of the Ras/MEK/ERK signaling pathway," Molecular Biology Reports, vol. 39, no. 6, pp. 6857-6866, 2012.

[35] V. Gianturco, A. Bellomo, E. D'ottavio et al., "Impact of therapy with $\alpha$-lipoic acid (ala) on the oxidative stress in the controlled niddm: a possible preventive way against the organ dysfunction?," Archives of Gerontology and Geriatrics, vol. 49, Supplement 1, pp. 129-133, 2009.

[36] A. K. Sabharwal and J. M. May, "alpha-Lipoic acid and ascorbate prevent LDL oxidation and oxidant stress in endothelial cells," Molecular and Cellular Biochemistry, vol. 309, no. 1-2, pp. 125-132, 2008.

[37] Y. Zhang, P. Han, N. Wu et al., "Amelioration of lipid abnormalities by $\alpha$-lipoic acid through antioxidative and antiinflammatory effects," Obesity, vol. 19, no. 8, pp. 1647-1653, 2011.

[38] S. Sun, M. Zhang, Q. Yang et al., "Resveratrol suppresses lipoprotein-associated phospholipase A2expression by reducing oxidative stress in macrophages and animal models," Molecular Nutrition \& Food Research, vol. 61, no. 10, 2017.

[39] I. B. Asztalos, J. A. Gleason, S. Sever et al., "Effects of eicosapentaenoic acid and docosahexaenoic acid on cardiovascular disease risk factors: a randomized clinical trial," Metabolism: Clinical and Experimental, vol. 65, no. 11, pp. 1636-1645, 2016.

[40] D. Matthews, J. Hosker, A. Rudenski, B. Naylor, D. Treacher, and R. Turner, "Homeostasis model assessment: insulin resistance and $\beta$-cell function from fasting plasma glucose and insulin concentrations in man," Diabetologia, vol. 28, no. 7, pp. 412-419, 1985.

[41] M. Okazaki, K. Sasamoto, T. Muramatsu, and S. Hosaki, "Evaluation of precipitation and direct methods for HDLcholesterol assay by HPLC," Clinical Chemistry, vol. 43, no. 10, pp. 1885-1890, 1997.

[42] E. de Juan-Franco, A. Pérez, V. Ribas et al., "Standardization of a method to evaluate the antioxidant capacity of high-density lipoproteins," International Journal of Biomedical Science: IJBS, vol. 5, no. 4, pp. 402-410, 2009.

[43] C. Craig, A. Marshall, M. Sjöström et al., "International physical activity questionnaire: 12-country reliability and validity," Medicine \& Science in Sports \& Exercise, vol. 35, no. 8, pp. 1381-1395, 2003.

[44] M. B. Moghaddam, F. B. Aghdam, M. A. Jafarabadi, H. Allahverdipour, S. D. Nikookheslat, and S. Safarpour, "The Iranian Version of International Physical Activity Questionnaire (IPAQ) in Iran: content and construct validity, factor structure, internal consistency and stability," World Applied Scienctific Journal, vol. 18, no. 8, pp. 1073-1080, 2012.

[45] C. Forde, Scoring the international physical activity questionnaire (IPAQ), University of Dublin, 2018.

[46] N. Namazi, B. Larijani, and L. Azadbakht, "Alpha-lipoic acid supplement in obesity treatment: a systematic review and meta-analysis of clinical trials," Clinical Nutrition, vol. 37, no. 2, pp. 419-428, 2018.

[47] K. P. Shay, R. F. Moreau, E. J. Smith, A. R. Smith, and T. M. Hagen, "Alpha-lipoic acid as a dietary supplement: molecular mechanisms and therapeutic potential," Biochimica et Biophysica Acta (BBA)-General Subjects, vol. 1790, no. 10, pp. 11491160, 2009.

[48] S. Lavi, R. Lavi, J. P. McConnell, L. O. Lerman, and A. Lerman, "Lipoprotein-associated phospholipase $\mathrm{A}_{2}$ : review of its role as a marker and a potential participant in coronary endothelial 
dysfunction," Molecular Diagnosis \& Therapy, vol. 11, no. 4, pp. 219-226, 2007.

[49] W.-Y. Wang, J. Li, D. Yang, W. Xu, R.-p. Zha, and Y.-p. Wang, "OxLDL stimulates lipoprotein-associated phospholipase $\mathrm{A}_{2}$ expression in THP-1 monocytes via PI3K and p38 MAPK pathways," Cardiovascular Research, vol. 85 , no. 4, pp. 845852, 2010.

[50] Y. Shi, P. Zhang, L. F. Zhang et al., "Role of lipoproteinassociated phospholipase $\mathrm{A}_{2}$ in leukocyte activation and inflammatory responses," Atherosclerosis, vol. 191, no. 1, pp. 54-62, 2007.

[51] Lp-PLA2 Studies Collaboration, A. Thompson, P. Gao et al., "Lipoprotein-associated phospholipase $\mathrm{A}_{2}$ and risk of coronary disease, stroke, and mortality: collaborative analysis of 32 prospective studies," The Lancet, vol. 375, no. 9725, pp. 1536-1544, 2010.

[52] L. Yang, Y. Liu, S. Wang, T. Liu, and H. Cong, "Association between Lp-PLA2 and coronary heart disease in Chinese patients," Journal of International Medical Research, vol. 45, no. 1, pp. 159-169, 2017.

[53] M. Kim, M. Kim, A. Lee et al., "Impact of 8-week linoleic acid intake in soy oil on Lp-PLA2 activity in healthy adults," Nutrition \& Metabolism, vol. 14, no. 1, 2017.

[54] N. Hermans, A. van der Auwera, A. Breynaert et al., "A red yeast rice-olive extract supplement reduces biomarkers of oxidative stress, OxLDL and Lp-PLA $A_{2}$, in subjects with metabolic syndrome: a randomised, double-blind, placebo-controlled trial," Trials, vol. 18, no. 1, p. 302, 2017.

[55] M. Kim, M. Kim, Y. J. Lee et al., "Effects of $\alpha$-linolenic acid supplementation in perilla oil on collagen- epinephrine closure time, activated partial thromboplastin time and Lp- PLA activity in non-diabetic and hypercholesterolaemic subjects," Journal of Functional Foods, vol. 23, pp. 95-104, 2016.

[56] M. Nakhjavani, O. Khalilzadeh, L. Khajeali et al., "Serum oxidized-LDL is associated with diabetes duration independent of maintaining optimized levels of LDL-cholesterol," Lipids, vol. 45, no. 4, pp. 321-327, 2010.

[57] J. K. Lodge, M. G. Traber, and L. Packer, "Thiol chelation of $\mathrm{Cu}^{2}$ by dihydrolipoic acid prevents human low density lipoprotein peroxidation," Free Radical Biology and Medicine, vol. 25, no. 3, pp. 287-297, 1998.

[58] C. J. Li, L. Lv, H. Li, and D. M. Yu, "Cardiac fibrosis and dysfunction in experimental diabetic cardiomyopathy are ameliorated by alpha-lipoic acid," Cardiovascular Diabetology, vol. 11, no. 1, p. 73, 2012.

[59] P. Chang, J. Liu, Y. Yu et al., "Alpha-lipoic acid suppresses extracellular histone-induced release of the infammatory mediator tumor necrosis factor- $\alpha$ by macrophages," Cellular Physiology and Biochemistry, vol. 42, no. 6, pp. 2559-2568, 2017.

[60] Z. Cavdar, M. A. Oktan, C. Ural et al., "Renoprotective effects of alpha lipoic acid on iron overload-induced kidney injury in rats by suppressing NADPH oxidase 4 and p38 MAPK signaling," Biological Trace Element Research, vol. 193, no. 2, pp. 483-493, 2020.

[61] L. S. Rallidis, C. C. Tellis, J. Lekakis et al., "Lipoprotein-associated phospholipase $\mathrm{A}_{2}$ bound on high-density lipoprotein is associated with lower risk for cardiac death in stable coronary artery disease patients: a 3-year follow-up," Journal of the American College of Cardiology, vol. 60, no. 20, pp. 20532060, 2012.
[62] The STABILITY Investigators, "Darapladib for preventing ischemic events in stable coronary heart disease," New England Journal of Medicine, vol. 370, no. 18, pp. 1702-1711, 2014.

[63] L. Wallentin, C. Held, P. W. Armstrong et al., "Lipoproteinassociated phospholipase A2 activity is a marker of risk but not a useful target for treatment in patients with stable coronary heart disease," Journal of the American Heart Association, vol. 5, no. 6, 2016.

[64] M. L. O’Donoghue, E. Braunwald, H. D. White et al., "Effect of darapladib on major coronary events after an acute coronary syndrome: the SOLID-TIMI 52 randomized clinical trial," Journal of the American Medical Association, vol. 312, no. 10, pp. 1006-1015, 2014.

[65] C. Schliefsteiner, B. Hirschmugl, S. Kopp et al., "Maternal gestational diabetes mellitus increases placental and foetal lipoprotein-associated phospholipase A2 which might exert protective functions against oxidative stress," Scientific Reports, vol. 7, no. 1, article 12628, 2017.

[66] J.-C. Fruchart, "Peroxisome proliferator-activated receptor- $\alpha$ activation and high-density lipoprotein metabolism," The American Journal of Cardiology, vol. 88, no. 12, pp. 24-29, 2001.

[67] J.-B. Wang, Y.-J. Zhang, Y. Zhang et al., "Negative correlation between serum syndecan-1 and apolipoprotein A1 in patients with type 2 diabetes mellitus," Acta Diabetologica, vol. 50, no. 2, pp. 111-115, 2013.

[68] B. De Geest, D. Stengel, M. Landeloos et al., "Effect of overexpression of human apo A-I in C57BL/6 and C57BL/6 apo Edeficient mice on 2 lipoprotein-associated enzymes, plateletactivating factor acetylhydrolase and Paraoxonase," Arteriosclerosis, Thrombosis, and Vascular Biology, vol. 20, no. 10, pp. E68-E75, 2000.

[69] T. Kujiraoka, T. Iwasaki, M. Ishihara et al., "Altered distribution of plasma PAF-AH between HDLs and other lipoproteins in hyperlipidemia and diabetes mellitus," Journal of Lipid Research, vol. 44, no. 10, pp. 2006-2014, 2003.

[70] S. Golbidi, M. Badran, and I. Laher, "Diabetes and alpha lipoic acid," Frontiers in Pharmacology, vol. 2, p. 69, 2011.

[71] M. B. Gomes and C. A. Negrato, "Alpha-lipoic acid as a pleiotropic compound with potential therapeutic use in diabetes and other chronic diseases," Diabetology \& Metabolic Syndrome, vol. 6, no. 1, p. 80, 2014.

[72] A. M. de Oliveira, P. H. C. Rondó, L. A. Luzia, F. H. D’Abronzo, and V. K. Illison, "The effects of lipoic acid and $\alpha$ tocopherol supplementation on the lipid profile and insulin sensitivity of patients with type 2 diabetes mellitus: a randomized, double-blind, placebo-controlled trial," Diabetes Research and Clinical Practice, vol. 92, no. 2, pp. 253-260, 2011.

[73] V. M. Mendoza-Núñez, B. I. García-Martínez, J. Rosado-Pérez, E. Santiago-Osorio, J. Pedraza-Chaverri, and V. J. HernándezAbad, "The effect of $600 \mathrm{mg}$ alpha-lipoic acid supplementation on oxidative stress, inflammation, and RAGE in older adults with type 2 diabetes mellitus," Oxidative Medicine and Cellular Longevity, vol. 2019, Article ID 3276958, 12 pages, 2019.

[74] H. Ansar, Z. Mazloom, F. Kazemi, and N. Hejazi, "Effect of alpha-lipoic acid on blood glucose, insulin resistance and glutathione peroxidase of type 2 diabetic patients," Saudi Medical Journal, vol. 32, no. 6, pp. 584-588, 2011.

[75] S. M. Mousavi, S. Shab-Bidar, H. Kord-Varkaneh, M. Khorshidi, and K. Djafarian, "Effect of alpha-lipoic acid supplementation on lipid profile: a systematic review and meta-analysis of controlled clinical trials," Nutrition, vol. 59, pp. 121-130, 2019. 